\section{CONSERVATIVE DENTISTRY}

\section{5-year follow-up of a prospective clinical study on various types of core restorations}

Creugers NHJ, Mentink AGM et al Int J Prosthodont 2005; 18:34-39

Type of post and core did not influence restoration survival, but remaining dentine height after preparation did.

In this study, 127 cast palladium posts and cores were compared with 150 direct posts and cores and 42 all composite cores without posts. Patients had one or more endodontically-treated teeth needing crowns. The posts and cores were placed by 18 operators. The minimum height of dentine was prescribed for the trial as $<75 \% 1 \mathrm{~mm}$ above gingival level and $>25 \%$ less than $1 \mathrm{~mm}$ above, or without a collar of 1-2 mm.

During the study, there were 5 early failures and 10 later ones. The early failures were all root fractures originating from 2 operators. There were no significant differences between the 3 types of restoration, but survival was significantly better in teeth with substantial dentine height $(>75 \%$ of minimum $1 \mathrm{~mm}$ thickness with a height of at least $1 \mathrm{~mm}$ above gingival level, and a collar of 1-2 mm) than in those with minimal height.

doi:10.1038/sj.bdj.4812527

\section{MAXILLOFACIAL SURGERY; OPHTHALMOLOGY}

Blindness after facial fractures: a 19-year retrospective study

Ansari MH J Oral Maxillofac Surg 2005; 63: 229-237

Laterally directed traumatic forces appeared to be significant factors affecting vision.

In an Iranian hospital serving a population of about 2 million people, 2,503 patients were admitted with facial fractures over a 19 year period. Ocular injuries were found in 83, severe visual impairment in 9 and blindness in 30. In 19 of the blindness group, the cause was a road traffic accident. The left eye was involved in 19 cases and the right eye in 11. Twenty-six cases were in men.

Loss of vision was in 23 cases associated with fracture of the zygoma or zygomaxillary complex, while 5 resulted from Le Fort III and 1 each from Le Fort II and nasoethmoid frontal trauma. Nearly half (14) of blindness cases were associated with retrobulbar haemorrhage, and a further 11 with complete eye damage. The authors recommend a complete ophthalmological examination before treatment of any facial fractures in cases where blindness is a presenting symptom.

\section{CONSERVATIVE DENTISTRY}

\section{Reasons for restorative therapy and the longevity of restorations in adults}

Forss H, Widström E Acta Odont Scand 2004; 62: 82-86

Most restorations placed by a large sample of Finnish dentists were replacements of previous restorations.

In this study, 548 of a random sample of 800 dentists responded to a request for data on restorations placed in 1 working day. Those who had not done such work $(n=68)$ were excluded, and the final response rate was 66\%. Altogether, 3,455 restorations were placed in 2,748 patients of mean age 52 yrs (range 17-94).

Composite resin was used in $79 \%$ of restorations, amalgam in $5 \%$, glass ionomer in 7\%, compomers in $4 \%$ and indirect methods in $5 \%$. If the reason for treatment was tooth or restoration fracture, restoration loss or marginal leakage, composite was more likely to be used. Glass ionomers and compomers were more likely to be used in young patients. In younger patients, primary caries was more often the reason for treatment.

The authors note that although composite resin was the commonest restorative material, there was no indication of patients wanting satisfactory amalgams replaced.

doi:10.1038/sj.bdj.4812529

\section{DENTAL RADIOLOGY; NEPHROLOGY}

\section{The prevalence of carotid artery} calcification on the panoramic radiographs of patients with renal disease

Kansu Ö, Özbek M atal Dentomaxillofac Radiol 2005; 34: 16-19

There was more carotid artery calcification (CAC) in dialysis and transplant patients.

Early detection of carotid artery calcification may help to identify patients at risk of severe cardiovascular problems. In this study, 34 patients on haemodialysis (HD) and 35 renal transplant recipients (RT) were compared with 50 healthy matched controls (C). All radiographs were evaluated twice by 3 experts.

In HD subjects, 2 unilateral and 5 bilateral CACs were identified, in RT subjects, 1 unilateral and 5 bilateral CACs, and in C subjects, 3 unilateral CACs $(P<0.005)$. CACs were observed as heterogeneous radiopacities at $\mathrm{C} 3-\mathrm{C} 4$ level.

Whilst panoramic radiographs are not a complete screening tool for these calcifications, the authors suggest that all such radiographs should be carefully examined for evidence of CACs. They note a number of other calcified bodies which may appear on radiographs in the same region of the neck.

doi:10.1038/sj.bdj.4812530 